Homology, Homotopy and Applications, vol. 20(1), 2018, pp.1-4

\title{
$K$-THEORETIC TORSORS FOR INFINITE DIMENSIONAL VECTOR BUNDLES OF LOCALLY COMPACT TYPE
}

\author{
SHO SAITO \\ (communicated by Charles A. Weibel)
}

\begin{abstract}
Drinfeld observed that there were apparently two notions of $K$-theory torsor one might expect to associate to a Tate $R$ module, and that these should be equivalent. The purpose of the present note is to explain this equivalence as a direct consequence of the author's delooping theorem and Drinfeld's theorem that the first negative $K$-group vanishes Nisnevich locally.
\end{abstract}

\section{Introduction}

Drinfeld observed in [3] that there were apparently two notions of $K$-theory torsor one might expect to associate to a topological module called a Tate $R$-module, and that these should be equivalent. The purpose of the present note is to explain this equivalence as a direct consequence of the author's delooping theorem of $[8]$ and Drinfeld's theorem of [3] that the first negative $K$-group vanishes Nisnevich locally.

Let $S$ be a quasi-compact, quasi-separated noetherian scheme of finite Krull dimension with an ample family of line bundles. We denote by $\operatorname{Shv}_{\text {(Spaces) }}\left(N S_{\mathrm{Nis}}\right)$ the $\infty$ topos of sheaves of spaces on the small Nisnevich site $S_{\text {Nis }}$ of $S$; by $\mathcal{K}$ the sheaf of $K$-theory spaces $S^{\prime} \mapsto \Omega^{\infty} K\left(S^{\prime}\right)$ on $S_{\text {Nis }}$ (here $K(-)$ is the connective $K$-theory spectrum) regarded as a group object in $\operatorname{Shv}_{\text {(Spaces) }}\left(N S_{\mathrm{Nis}}\right)$; and by $B \mathcal{K}$ the classifying space for $\mathcal{K}$-torsors. (See section 2 below for more details.)

Recall the construction $\lim$ introduced in [1, A.3], that associates to an exact category $\mathcal{A}$ a new one $\lim _{\longleftrightarrow} \overleftrightarrow{\mathcal{A} \subset}$ Ind $\operatorname{Pro}(\mathcal{A})$. We write $\operatorname{Vect}_{\text {Tate }}(S)$ for the idempotent completion of $\lim \operatorname{Vect}_{\text {finite }}(S)$, where $\operatorname{Vect}_{\text {finite }}(S)$ is the exact category of finite-rank vector bundles. If $S=\operatorname{Spec} R$ is affine, $\operatorname{Vect}_{\text {Tate }}(S)$ embeds into the category of Tate $R$-modules in the sense of [3], being equivalent to the full subcategory of Tate $R$ modules of countable type; see [2, Theorem 5.30] for details. Denote by $\mathcal{K}_{\text {Tate }}$ the sheaf of spaces $S^{\prime} \mapsto \Omega^{\infty} \mathbb{K}\left(\operatorname{Vect}\right.$ Tate $\left.\left(S^{\prime}\right)\right)$ on $S_{\text {Nis }}$, where $\mathbb{K}(-)$ denotes the non-connective $K$-theory spectrum. (See section 2 below for more details.)

In this short note, we prove the following results.

Theorem 1.1. There is an equivalence $B \mathcal{K} \stackrel{\sim}{\longrightarrow} \mathcal{K}_{\text {Tate }}$ in the $\infty$-topos $\mathrm{Shv}_{\text {(Spaces) }}\left(N S_{\mathrm{Nis}}\right)$.

This work was supported by the WPI initiative, MEXT, Japan.

Received February 15, 2017, revised May 26, 2017; published on December 13, 2017.

2010 Mathematics Subject Classification: 19E99.

Key words and phrases: $K$-theoretic torsor, Tate $R$-module.

Article available at http://dx.doi.org/10.4310/HHA.2018.v20.n1.a1

Copyright (C) 2017, International Press. Permission to copy for private use granted. 
Let $\mathcal{E}$ be an object of $\operatorname{Vect}_{\text {Tate }}(S)$ and $[\mathcal{E}] \in \mathcal{K}_{\text {Tate }}(S)=\Omega^{\infty} \mathbb{K}\left(\operatorname{Vect}_{\text {Tate }}(S)\right)$ the associated point in the $K$-theory space. The map $S \stackrel{[\mathcal{E}]}{\rightarrow} \mathcal{K}_{\text {Tate }}$ corresponds by the above theorem to a $\mathcal{K}$-torsor $\mathfrak{D}_{\mathcal{E}}$ on $S$. Write $\operatorname{Aut}(\mathcal{E})$ for the sheaf of groups on $S_{\text {Nis }}$ given by $S^{\prime} \mapsto \operatorname{Aut}_{\text {Vect Tate }}\left(S^{\prime}\right) \mathcal{E} \times_{S} S^{\prime}$. This is a group object of the 1 -topos $\operatorname{Shv}(\operatorname{Sets})\left(S_{\mathrm{Nis}}\right)$, and hence of the $\infty$-topos $\operatorname{Shv}_{\text {(Spaces) }}\left(N S_{\text {Nis }}\right) \supset \operatorname{Shv}_{(\text {Sets })}\left(S_{\text {Nis }}\right)$.

Theorem 1.2. The automorphism group Aut $\mathcal{E}$ acts on the $\mathcal{K}$-torsor $\mathfrak{D}_{\mathcal{E}}$.

Remark 1.3. Considering the composite map $S \stackrel{[\mathcal{E}]}{\rightarrow} \mathcal{K}_{\text {Tate }}=B \mathcal{K} \rightarrow B \tau_{\leqslant 1} \mathcal{K}$ instead of $[\mathcal{E}]$ itself (where $B \mathcal{K} \rightarrow B \tau_{\leqslant 1} \mathcal{K}$ is induced from the truncation map), we get a $\tau_{\leqslant 1} \mathcal{K}$ torsor $D_{\mathcal{E}}$. The proof of Theorem 1.2 shows Aut $\mathcal{E}$ acts on $D_{\mathcal{E}}$ as well, defining an enriched $\mathbb{G}_{m}$-central extension of Aut $\mathcal{E}$. Cf. [3, section 5.5].

\section{2. $\infty$-topos formalism}

In this preliminary section, we give a detailed account on the notions and objects involved in Theorems 1.1 and 1.2, in order to ensure that the statements make sense to the reader even if he or she is unfamiliar with the language of $\infty$-topoi.

Write $\operatorname{Set}_{\Delta}$ for the category of simplicial sets, which is a combinatorial, simplicial model category with the Kan model structure. Recall that $S_{\text {Nis }}$ denotes the small Nisnevich site of the scheme $S$. We write $\operatorname{Set}_{\Delta}^{S_{\mathrm{Nis}}^{\mathrm{op}}}$ for the combinatorial, simplicial model category of simplicial presheaves on the underlying category of $S_{\text {Nis }}$ with the injective model structure, and $\left(\operatorname{Set}_{\Delta}^{S_{\mathrm{Nis}}^{\mathrm{op}}}\right)^{\circ}$ for its fibrant-cofibrant objects. By Proposition 4.2.4.4 of [5], there is an equivalence of $\infty$-categories

$$
\theta: N\left(\operatorname{Set}_{\Delta}^{S_{\mathrm{Nis}}^{\text {op }}}\right)^{\circ} \stackrel{\sim}{\rightarrow} \operatorname{Fun}\left(N S_{\mathrm{Nis}}^{\mathrm{op}},(\text { Spaces })\right)=\operatorname{Preshv}_{(\text {Spaces })}\left(N S_{\mathrm{Nis}}\right) .
$$

Here $N$ denotes the simplicial nerve, $\operatorname{Fun}(-,-)$ the $\infty$-category of functors, (Spaces) the $\infty$-category of spaces, which is by definition the simplicial nerve of the simplicial category of Kan complexes, and $\operatorname{Preshv}_{\text {(Spaces) }}\left(N S_{\mathrm{Nis}}\right)$ the $\infty$-category of presheaves of spaces on $N S_{\text {Nis }}$. Let $\operatorname{Set}_{\Delta \text {,loc }}^{S_{\text {Nis }}^{\text {op }}}$ denote the combinatorial, simplicial model category of simplicial presheaves on the site $S_{\mathrm{Nis}}$ with respect to Jardine's local model structure [4], and $\left(\operatorname{Set}_{\Delta, \text { loc }}^{S_{\mathrm{Nis}}}\right)^{\circ}$ its fibrant-cofibrant objects. Then Proposition 6.5.2.14 of [5] shows that the above equivalence $\theta$ restricts to an equivalence

$$
\theta: N\left(\operatorname{Set}_{\Delta, \text { loc }}^{S_{\mathrm{Nis}}^{\text {op }}}\right)^{\circ} \stackrel{\sim}{\rightarrow} \operatorname{Shv}_{\text {(Spaces) }}\left(N S_{\text {Nis }}\right)^{\wedge} \stackrel{\sim}{\hookrightarrow} \operatorname{Shv}_{\text {(Spaces) }}\left(N S_{\text {Nis }}\right),
$$

where $\operatorname{Shv}_{\text {(Spaces) }}\left(N S_{\mathrm{Nis}}\right) \subset \operatorname{Preshv}_{\text {(Spaces) }}\left(N S_{\mathrm{Nis}}\right)$ is the $\infty$-topos of sheaves of spaces on $N S_{\mathrm{Nis}}\left[\mathbf{5}, 6.1 .0 .4\right.$ and 6.2.2], and $\operatorname{Shv}_{\text {(Spaces) }}\left(N S_{\mathrm{Nis}}\right)^{\wedge}$ denotes its hypercompletion $[\mathbf{5}, 6.5 .2]$, with the inclusion functor $\operatorname{Shv}_{\text {(Spaces) }}\left(N S_{\text {Nis }}\right)^{\wedge} \stackrel{\sim}{\hookrightarrow} \operatorname{Shv}_{(\text {Spaces })}\left(N S_{\text {Nis }}\right)$ being an equivalence by $[\mathbf{6}, 2.25]$, since $S$ is noetherian and of finite Krull dimension.

Consider the simplicial presheaf of $K$-theory spaces $S^{\prime} \mapsto \Omega^{\infty} K\left(S^{\prime}\right)$ on the Nisnevich site $S_{\mathrm{Nis}}$, where $K\left(S^{\prime}\right)$ is the connective $K$-theory spectrum. Since the connective and non-connective $K$-theory spectra of a scheme have the same non-negative homotopy groups, the canonical map $K(-) \rightarrow \mathbb{K}(-)$ induces a weak equivalence $\Omega^{\infty} K(-) \stackrel{\sim}{\rightarrow} \Omega^{\infty} \mathbb{K}(-)$ of simplicial presheaves in $\operatorname{Set}_{\Delta}^{S_{\mathrm{Nis}}^{\mathrm{op}}}$, where $\mathbb{K}$ denotes the nonconnective $K$-theory spectrum. By Thomason's theorem, non-connective $K$-theory 
satisfies Nisnevich descent. It follows that the simplicial presheaf $\Omega^{\infty} K(-)$ is a fibrant object of $\operatorname{Set}_{\Delta, \text { loc }}^{S_{\mathrm{Ni}}^{\mathrm{op}}}$, so that it defines an object of the $\infty$-topos $\operatorname{Shv}_{\text {(Spaces) }}\left(N S_{\mathrm{Nis}}\right)$ by the above equivalence $\theta$. We write $\mathcal{K}$ for this object $\theta\left(\Omega^{\infty} K(-)\right)$ and call it the sheaf of $K$-theory spaces on $S$.

Since the $K$-theory space is the infinite loop space associated to a connective spectrum, $\mathcal{K}$ has a structure of a group object in $\operatorname{Shv}_{\text {(Spaces) }}\left(N S_{\mathrm{Nis}}\right)$. (More precisely, by $[\mathbf{5}, 7.2 .2 .11-(1)]$, to give a group structure on an object $G$ of an $\infty$-topos $\mathfrak{X}$ is equivalent to give a locally connected delooping $B G$ of $G$. Consider the simplicial presheaf $S^{\prime} \mapsto \Omega^{\infty} \Sigma K\left(S^{\prime}\right)$ on $S_{\mathrm{Nis}}$ and its image $\theta\left(\Omega^{\infty} \Sigma K(-)\right)$ in the $\infty$-category $\operatorname{Preshv}_{\text {(Spaces) }}\left(N S_{\mathrm{Nis}}\right)$ under the equivalence $\theta$. The sheafification $a\left(\theta\left(\Omega^{\infty} \Sigma K(-)\right)\right) \in$ ob $\operatorname{Shv}_{\text {(Spaces) }}\left(N S_{\text {Nis }}\right)$ of $\theta\left(\Omega^{\infty} \Sigma K(-)\right)$ serves as a locally connected delooping of $\mathcal{K}$.) In particular, there is a notion of $\mathcal{K}$-torsor in $\operatorname{Shv}_{\text {(Spaces) }}\left(N S_{\text {Nis }}\right)$, as a particular case of the notion of $G$-torsor for a general group object $G$ defined in [7, Definition 3.4], and we have the classifying space $B \mathcal{K}$ that classifies $\mathcal{K}$-torsors in the sense that for every object $\mathcal{F}$ of $\operatorname{Shv}_{\text {(Spaces) }}\left(N S_{\text {Nis }}\right)$ the $\infty$-groupoid of $\mathcal{K}$-torsors on $\mathcal{F}$ is equivalent to the mapping space $\operatorname{Map}_{\mathrm{Shv}_{(\text {Spaces })}\left(N S_{\mathrm{Nis}}\right)}(\mathcal{F}, B \mathcal{K})$.

Recall that $\operatorname{Vect}_{\text {Tate }}(S)$ denotes the idempotent completion of $\lim _{\longleftrightarrow} \operatorname{Vect}_{\text {finite }}(S)$, where $\operatorname{Vect}_{\text {finite }}(S)$ is the exact category of finite-rank vector bundles on $S$ and $\lim$ is the construction on exact categories introduced in [1, A.3]. By Theorem 1.2 of $[\mathbf{8}]$, there is an equivalence $\Sigma \mathbb{K}(S) \stackrel{\sim}{\rightarrow} \mathbb{K}\left(\operatorname{Vect}_{\text {Tate }}(S)\right)$. The presheaf of spectra on $S_{\text {Nis }}$ given by $S^{\prime} \mapsto \Sigma \mathbb{K}\left(S^{\prime}\right)=\mathbb{K}\left(\operatorname{Vect}_{\text {Tate }}\left(S^{\prime}\right)\right)$ satisfies Nisnevich descent by Thomason's theorem, and hence the simplicial presheaf on $S_{\text {Nis }}$ given by $S^{\prime} \mapsto \Omega^{\infty} \mathbb{K}\left(\operatorname{Vect}_{\text {Tate }}\left(S^{\prime}\right)\right)$ is a fibrant object of $\operatorname{Set}_{\Delta \text {,loc }} S_{\mathrm{Nis}}^{\mathrm{op}}$, and thus defines via the equivalence $\theta$ an object of the $\infty$-topos $\operatorname{Shv}_{(\text {Spaces }}\left(N S_{\text {Nis }}\right)$. We write $\mathcal{K}_{\text {Tate }}$ for this object $\theta\left(\Omega^{\infty} \mathbb{K}\left(\operatorname{Vect}_{\text {Tate }}(-)\right)\right)$.

\section{Proofs}

Recall:

Theorem 3.1 ([8], Theorem 1.2). There is a natural equivalence of spectra $\Sigma \mathbb{K}\left(S^{\prime}\right) \stackrel{\sim}{\rightarrow}$ $\mathbb{K}\left(\operatorname{Vect}_{\text {Tate }}\left(S^{\prime}\right)\right)$ for every object $S^{\prime}$ in $S_{\text {Nis }}$.

Theorem 3.2 ([3], Theorem 3.7). Every element of the first negative K-group vanishes Nisnevich locally. I.e., the sheafification of the presheaf $S^{\prime} \mapsto K_{-1}\left(S^{\prime}\right)$ on $S_{\mathrm{Nis}}$ is trivial.

Theorem 3.3 ([7], Theorem 3.17). Let $\mathfrak{X}$ be a general $\infty$-topos and $G$ a group object. Then the classifying space $B G$ for $G$-torsors is equivalent to the locally connected delooping of $G$.

Proof of Theorem 1.1. By Theorem 3.1, the object $\mathcal{K}_{\text {Tate }}$ is a delooping of $\mathcal{K}$, and is locally connected by Theorem 3.2. Theorem 3.3 now tells that $\mathcal{K}_{\text {Tate }}$ is equivalent to the classifying space $B \mathcal{K}$, proving Theorem 1.1 .

Recall:

Proposition 3.4 (See [7], section 3). Let $\mathfrak{X}$ be a general $\infty$-topos and $G$ a group object. Then, giving a G-action on an object $P$ of $\mathfrak{X}$ is equivalent to giving a fiber sequence $P \rightarrow X \rightarrow B G$ for some object $X$. 
Proof of Theorem 1.2. By Proposition 3.4, constructing the desired Aut $(\mathcal{E})$-action on the $\mathcal{K}$-torsor $\mathfrak{D}_{\mathcal{E}}$ amounts to describing $\mathfrak{D}_{\mathcal{E}}$ as a pullback $\mathfrak{D}_{\mathcal{E}}=\lim (X \rightarrow B \operatorname{Aut}(\mathcal{E}) \leftarrow$ $S$ ) for some $X$ and some map $X \rightarrow B \operatorname{Aut}(\mathcal{E})$. It turns out that it suffices to have a map $[[\mathcal{E}]]: B \operatorname{Aut}(\mathcal{E}) \rightarrow \mathcal{K}_{\text {Tate }}$ whose precomposition with the base-point map $S \rightarrow$ $B \operatorname{Aut}(\mathcal{E})$ is equivalent to the map $[\mathcal{E}]: S \rightarrow \mathcal{K}_{\text {Tate }}=B \mathcal{K}$ classifying the $\mathcal{K}$-torsor $\mathfrak{D}_{\mathcal{E}}$.

We first note that, in general, for an exact category $\mathcal{A}$ and an object $A$ of $\mathcal{A}$ the connected delooping $B$ Aut $_{\mathcal{A}} A$ of the group $\operatorname{Aut}_{\mathcal{A}} A$ admits a canonical map to the $K$-theory space $\Omega^{\infty} K(\mathcal{A})$ given as the composition $B$ Aut $_{\mathcal{A}} A \rightarrow N i \mathcal{A} \rightarrow \Omega i S \cdot \mathcal{A}=$ $\Omega^{\infty} K(\mathcal{A})$, where $N i \mathcal{A}$ denotes the nerve of the maximal groupoid of $\mathcal{A}$ and the latter arrow is the first structure map of the spectrum $K(\mathcal{A})$. We also remark that if the exact category $\mathcal{A}$ is idempotent complete then $\Omega^{\infty} K(\mathcal{A}) \stackrel{\sim}{\rightarrow} \Omega^{\infty} \mathbb{K}(\mathcal{A})$, and we thus get a map $B$ Aut $_{\mathcal{A}} A \rightarrow \Omega^{\infty} \mathbb{K}(\mathcal{A})$. In view of this we see that there is a map of simplicial presheaves $B$ Aut $_{\text {Vect Tate }(-)} \mathcal{E} \times_{S}(-) \rightarrow \Omega^{\infty} \mathbb{K}\left(\operatorname{Vect}_{\text {Tate }}(-)\right)$ in $\operatorname{Set}_{\Delta}^{S_{\mathrm{Nis}}^{\mathrm{op}}}$. It induces a $\operatorname{map}[[\mathcal{E}]]: a\left(\theta\left(B \operatorname{Aut}_{\text {Vect }_{\text {Tate }}(-)} \mathcal{E} \times_{S}(-)\right)\right)=B$ Aut $\mathcal{E} \rightarrow \mathcal{K}_{\text {Tate }}$ in $\operatorname{Shv}_{\text {(Spaces) }}\left(N S_{\text {Nis }}\right)$, where $a(-)$ denotes the sheafification. By construction, the precomposition of $[[\mathcal{E}]]$ with the base-point map $S \rightarrow B$ Aut $\mathcal{E}=a\left(\theta\left(B \operatorname{Aut}_{\text {Vect }_{\text {Tate }}(-)} \mathcal{E} \times_{S}(-)\right)\right)$ corresponds to the point $[\mathcal{E}] \in \mathcal{K}_{\text {Tate }}(S)$, and the proof of Theorem 1.2 is complete.

\section{References}

[1] A. Beilinson, How to glue perverse sheaves, in: K-Theory, Arithmetic and Geometry (Moscow, 1984), Lecture Notes in Math. 1289, Springer, 1987, pp. 42-51.

[2] O. Braunling, M. Groechenig, J. Wolfson, Tate objects in exact categories (with an appendix by Jan Stovicek and Jan Trlifaj), Mosc. Math. J. 16(3), 2016, 433504 .

[3] V. Drinfeld, Infinite-dimensional vector bundles in algebraic geometry (an introduction), in: The Unity of Mathematics, Progr. Math. 244, Birkhäuser, 2006, pp. 263-304.

[4] J.F. Jardine, Simplicial presheaves, J. Pure Appl. Algebra 47(1), 1987, 35-87.

[5] J. Lurie, Higher Topos Theory, Ann. of Math. Stud. 170, Princeton Univ. Press, 2009.

[6] J. Lurie, Derived Algebraic Geometry XI: Descent Theorems, available at http://www.math.harvard.edu/ lurie/.

[7] T. Nikolaus, U. Schreiber, D. Stevenson, Principal $\infty$-bundles-General theory, J. Homotopy Relat. Struct. 10(4), 2015, 749-801.

[8] S. Saito, On Previdi's delooping conjecture for K-theory, Algebra Number Theory $9(1), 2015,1-11$.

Sho Saito sho.saito@ipmu.jp

Kavli IPMU, The University of Tokyo, 5-1-5 Kashiwanoha, Kashiwa, 277-8583, Japan 